Hydrology and Earth System Sciences, 7(2), 151-161 (2003) C EGU

\title{
Root selection methods in flood analysis
}

\author{
Benoît Parmentier, James C.I. Dooge and Michael Bruen \\ Centre for Water Resources Research, University College Dublin, Earlsfort Terrace, Dublin 2, Ireland \\ Email for corresponding author:
}

\begin{abstract}
In the 1970s, de Laine developed a root-matching procedure for estimating unit hydrograph ordinates from estimates of the fast component of the total runoff from multiple storms. Later, Turner produced a root selection method which required only data from one storm event and was based on recognising a pattern typical of unit hydrograph roots. Both methods required direct runoff data, i.e. prior separation of the slow response. This paper introduces a further refinement, called root separation, which allows the estimation of both the unit hydrograph ordinates and the effective precipitation from the full discharge hydrograph. It is based on recognising and separating the quicker component of the response from the much slower components due to interflow and/or baseflow. The method analyses the z-transform roots of carefully selected segments of the full hydrograph. The root patterns of these separate segments tend to be dominated by either the fast response or the slow response. This paper shows how their respective time-scales can be distinguished with an accuracy sufficient for practical purposes. As an illustration, theoretical equations are derived for a conceptual rainfall-runoff system with the input split between fast and slow reservoirs in parallel. These are solved analytically to identify the reservoir constants and the input splitting parameter. The proposed method, called "root separation", avoids the subjective selection of rainfall roots in the Turner method as well as the subjective matching of roots in the original de Laine method.
\end{abstract}

Keywords: unit hydrograph, identification methods, z-transform, polynomial roots, root separation, fast and slow response, Nash cascade

\section{Introduction}

Difficulties often arise in relation to the estimation of effective precipitation for the purpose of flood prediction using linear models. These include (a) the difficulties in estimating areal precipitation accurately, (b) the uncertainties in relation to the initial soil moisture conditions in the catchment, (c) the reduction of total precipitation to effective precipitation due to infiltration and groundwater recharge. Errors in the unit-hydrograph derived from past storms due to such factors will affect the estimation of design floods or the performance of real-time flood forecasting systems. This situation suggests an investigation of the usefulness of those methods which have been developed to derive the unithydrograph without reference to rainfall data. Of particular interest in this connection is the root selection method (Turner et al., 1989)

This paper describes a theoretical study of this question as an adjunct to a study on the Dodder catchment. Numerical experiments were carried out using synthetic data based on simple arrangements of linear reservoirs to validate the methods and the computer program used against the corresponding analytical solution.

\section{The unit-hydrograph approach}

In this paper, direct runoff is taken to mean the total discharge at the catchment outlet minus the baseflow. The UnitHydrograph (UH) of a catchment can be defined as the hydrograph of direct runoff resulting from a fixed given pattern and defined volume of effective rainfall occurring over that catchment at a uniform rate during a unit period of time. The classical assumptions inherent in the UnitHydrograph method are given by Johnstone and Cross (1949). In essence, unit-hydrograph theory makes the mathematical assumptions that the system is linear and also time-invariant (Dooge, 1959). 
The relationship between the effective rainfall (input $x$ ) and the direct runoff (output $y$ ) can be expressed by the convolution integral:

$$
y(t)=\int_{0}^{t} h(t) x(\tau-t) \delta t
$$

where, $h(\mathrm{t})$ is the transfer function characterising the impulse response of the system, also called the instantaneous unit hydrograph. In practice, data are rarely analysed in continuous form. A discrete formulation of the convolution integral is (Dooge, 1973):

$$
y(n T)=\sum_{k=0}^{k=n} h(k T) x((n-k) T)
$$

where, $x(n T)$ is the volume of effective rainfall during the interval $t=n T$ to $t=(n+1) T$ and $y(n T)$ is the value of the direct runoff sampled at time $t=n T$. $h($.) represents the $T$ period unit hydrograph, i.e. the direct runoff due to unit volume of effective rainfall during an initial interval of duration $T$. This equation applies to isolated events. The standard estimation problem is to determine the values of the unit hydrograph ordinates, $h$, from measurements of the input series, $x$, and the output series, $y$. Eagleson et al. (1966) have shown that the convolution equation also relates the auto-correlation of the effective rainfall series and the crosscorrelation of the effective rainfall with the direct runoff series.

If there are $p$ output values, the discrete convolution equation gives $p$ simultaneous linear algebraic equations, which can, for convenience, be written in matrix form as:

$$
X h=y
$$

where:

- $\quad X$ is a $p \times n$ matrix formed from the $m$ input series values,

- $y$ is a vector containing the $p$ output series values,

- $\quad h$ is a vector representing the, as yet unknown, $n$ values of the pulse response.

The matrix $X$ has a particular structure. The number of columns is always less than the number of rows and is often considerably less. In ideal conditions:

$$
p=m+n-1
$$

In practice, because of data errors and uncertainty, it may be difficult to estimate the length of the unit hydrograph series.

\section{The Root-matching method (De Laine)}

The root matching method, based on runoff data only, was developed by de Laine $(1970,1975)$. It is based on finding the polynomial roots of the z-transform of the direct runoff of a number of isolated storms on a given catchment. The $\mathrm{z}$-transform, for any given discrete function, is defined as:

$$
F\left(z^{-1}\right)=\sum_{s=0}^{s=\infty} f(s T) z^{-s}
$$

where $F\left(z^{-1}\right)$ is the polynomial whose coefficients are the corresponding ordinates of the original function $f(s T)$.

For a linear time-invariant system, the z-transform of the input $X$, the pulse response $H$ and the output $Y$ are connected by:

$$
Y\left(z^{-1}\right)=X\left(z^{-1}\right) H\left(z^{-1}\right)
$$

where $Y, X$ and $H$ are respectively of order $p, m$ and $n$. Direct derivation of the transfer function by dividing the polynomials by the polynomial $X\left(z^{-1}\right)$ is not satisfactory because of the sensitivity of the result to errors in the data.

Knowing that a set of $p$ roots of is made up of the $m$ roots of $Y\left(z^{-1}\right)$ and the $n$ roots of $X\left(z^{-1}\right)$, de Laine(1970) suggested that, by examination of the outputs of two or more storms of differing characters, the common roots in the runoff polynomial could be identified as being independent of the rainfall and hence attributable only to the transfer function $h(s T)$. For this method, a compromise must be found regarding the frequency of sampling of the output. Indeed, the greater the frequency of sampling, the better the definition of the system. On the other hand, the more sampling points there are available, the more difficult it is to recognize pairs of roots. It is important to determine a suitable frequency of the sampling for any given catchment.

Four disadvantages of the basic root-matching method were identified by Turner et al. (1989). They can be summarised as follows:

1. The values of the complex roots for any given storm are very sensitive to the point of curtailment of the direct runoff hydrograph since the number and hence the value of the roots will vary with the duration of direct runoff assumed. The effect of this is to make root matching between two events very difficult.

2. The values of the roots are very sensitive to changes in the polynomial coefficients values, and hence the 
method is very sensitive to the quality of the runoff data.

3. The method of baseflow separation chosen can have a significant effect on the values of the complex roots. When the baseflow separation is inaccurate, the values of the complex roots will contain errors.

4. Common roots for the transfer function polynomials of several events can be found only if the catchment behaves as a perfect linear, time-invariant system. This is a strong assumption.

Turner et al. (1989) tested the de Laine method for a synthetic case based on assumed rainfall and runoff. The unit-hydrograph was reconstructed from the roots not related to the input polynomial. Numerical experiments indicated that a $10 \%$ error in the data would result in an average error of $7 \%$ in the unit hydrograph. That was a considerable improvement on the explosive error level of $5000 \%$ found when using polynomial division, but was still in excess of what could be achieved by using classical methods such as least-squares, orthogonal transforms or conceptual models Dooge (1979).

\section{The root selection method (Turner)}

Turner (1982) applied de Laine's method to a number of historical storm events for his study of reservoir inflows on the river Liffey. He plotted the resulting complex roots on an Argand diagram and noted several features for the different flood events:

- A definite relationship existed between the overall shapes of the root patterns for this particular catchment.

- A specific skewed circular pattern of roots was common to virtually all flood hydrographs.

Thus the transfer function and the effective rainfall can be identified, using only one flood event, by plotting the roots of the output (direct runoff) polynomial and by considering that:

- The roots associated with the transfer function appear either as points on a "skew circle" or as points on the real axis. The remaining roots are associated with the effective rainfall.

- By comparison with the de Laine method previously described, the Turner method also allows the analysis of longer flood events, since the pattern of the roots associated with the transfer function is more discernible on an Argand diagram.

To evaluate the significance of these observations, experiments were carried out in which the observed root patterns were compared with the root patterns obtained for conceptual models such as the Nash Cascade (Nash, 1958) commonly used to represent to the unit-hydrograph.

For the case of a single linear reservoir of delay time, K, and a sampling time, $\mathrm{T}$, the roots describe a circle of radius $\exp (T / K)$ centred on the origin. For the case of a Nash cascade of $n$ equal linear reservoirs, the root pattern of the polynomial plots as a 'skew circle' and the roots are regularly spaced.

Figures 1 and 2 show the unit-hydrographs for both a single reservoir $(K=5, n=1, N=20, T=1)$ and a Nash cascade of reservoirs $(K=1, n=5, N=20, T=1)$, together with their associated roots plotted on Argand diagrams.

The analogous discrete cascade model suggested by O'Connor $(1976,1982)$ will not be described here, as the
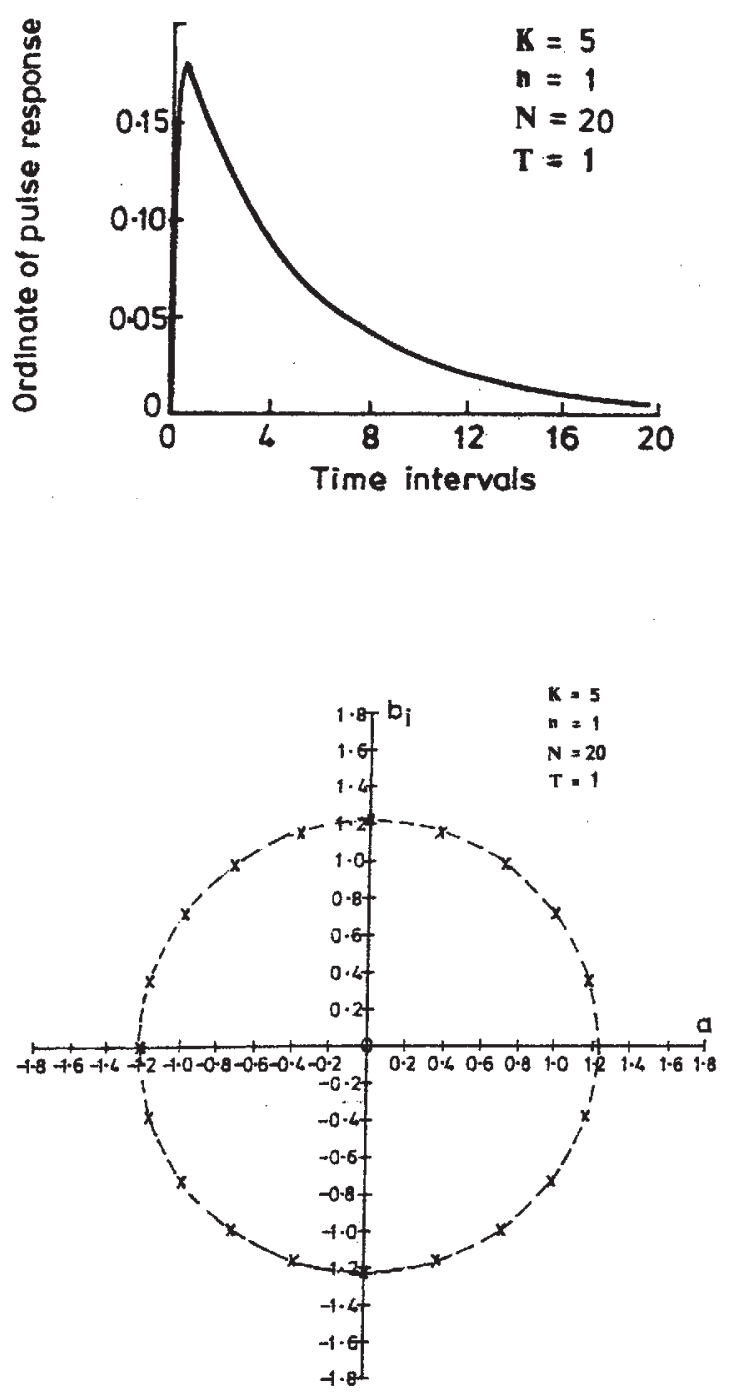

Fig. 1. Unit-hydrograph for a single reservoir and its associated $\operatorname{roots}(\mathrm{K}=5, \mathrm{n}=1, \mathrm{~N}=20, \mathrm{~T}=1)$ 

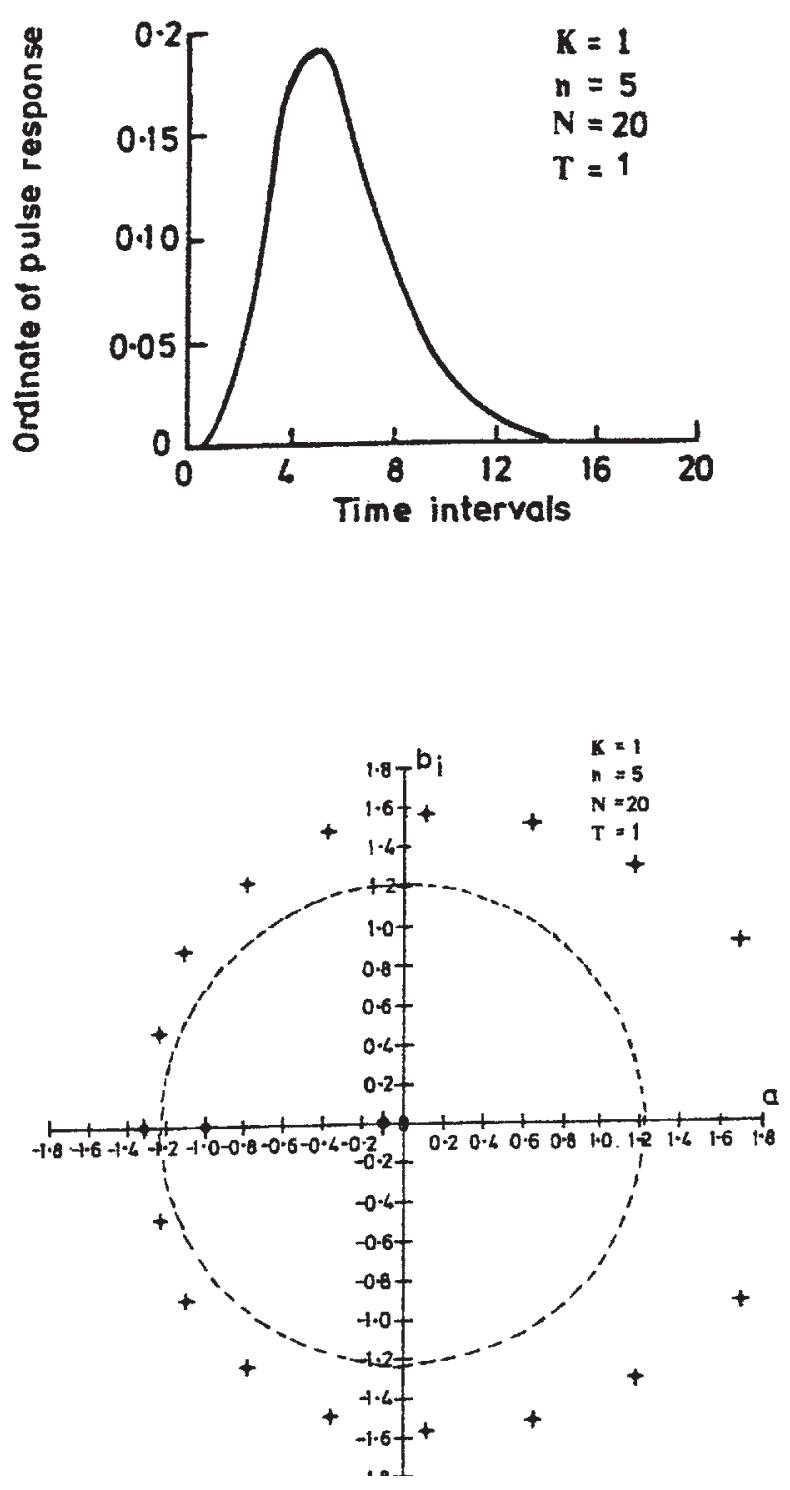

Fig. 2. Unit-hydrograph for a Nash cascade of 5 reservoirs and the associated $\operatorname{roots}(\mathrm{K}=1, \mathrm{n}=5, \mathrm{~N}=20, \mathrm{~T}=1)$

continuous Nash cascade model is concentrated on in developing a new approach as a variation on the Turner method. The latter assumed that there is a system response similitude between the natural catchment and the conceptual model of a cascade of equal reservoirs.

On the basis of worked examples, Turner (1982) has shown that the unit-hydrograph is largely or entirely made up of the roots on the skew circle, as would be expected considering the results for the conceptual models. When dealing with the 'skew circle', the selection or not of individual pairs of roots does not substantially alter the overall shape of the unit-hydrograph.

\section{Analysis of a 2-component response}

To improve on previous methods, the roots corresponding to the rainfall are separated and the total response is then split into a quick response and a slow response. The first step in the proposed method is to assume that the quick and slow responses can be represented by single linear reservoirs with time constants, $\mathrm{K}_{1}$ and $\mathrm{K}_{2}$ respectively; these are then estimated from the average radii of the root patterns.

The z-transform of the unit hydrograph for a single reservoir is given by:

$$
\begin{array}{r}
H\left(z^{-1}\right)=\left[\frac{\exp (T / K)-1}{T}\right] \exp (-T / k) z^{-1} \\
\left\{\frac{1-\left[\exp (-T / K) z^{-1}\right]^{N}}{1-\exp (-T / K) z^{-1}}\right\}
\end{array}
$$

For two reservoirs in parallel where a fraction, $\alpha$, of the flow goes through the fast reservoir with delay parameter $K_{1}$ and the remaining (1- $\left.\alpha\right)$ part of the flow goes through the slower reservoir with delay parameter $K_{2}(0 \leq \alpha \leq 1$ and $\left.K_{2}>K_{1}\right)$, the z-transform of the unit hydrograph is

$$
H\left(z^{-1}\right)=\alpha H_{1}\left(z^{-1}\right)+(1-\alpha) H_{2}\left(z^{-1}\right)
$$

By substituting Eqn. (7) twice into Eqn. (8) with the respective values of $K_{1}$ and $K_{2}$ in $H_{1}\left(z^{-1}\right)$ and $H_{2}\left(z^{-1}\right)$ the ztransform of the combined system can be derived as the ratio of two polynomials. Setting its numerator equal to zero, the root pattern for the two reservoirs in parallel is obtained.

Thus, the numerator of Eqn. (8) may be written as:

$$
\begin{array}{r}
\mathfrak{I}\left[H\left(z^{-1}\right)\right]=A_{1}\left\{1-\left[\exp \left(-T / K_{1}\right) z^{-1}\right]^{N}\right\}+ \\
A_{2}\left\{1-\left[\exp \left(-T / K_{2}\right) z^{-1}\right]^{N}\right\}
\end{array}
$$

where:

$$
\begin{array}{r}
A_{1}=\alpha\left[\exp \left(T / K_{1}\right)-1\right] \exp \left(-T / K_{1}\right) z^{-1} \\
{\left[1-\exp \left(-T / K_{2}\right) z^{-1}\right]}
\end{array}
$$

and

$$
\begin{aligned}
A_{2}=(1-\alpha)\left[\exp \left(T / K_{2}\right)-1\right] & \exp \left(-T / K_{2}\right) z^{-1} \\
& {\left[1-\exp \left(-T / K_{1}\right) z^{-1}\right] }
\end{aligned}
$$

Calculating the roots of the $z$-transform of the unit hydrograph requires setting the numerator, Eqn. (9), to zero and solving the resulting equations. Here, this is done numerically, using the method described by Jenkins and Traub (1972), and examining the negative real root values of $z^{-1}$. For the case of $T=1, K_{1}=5, K_{2}=20$ and various values of $\alpha$ from 0 to 1 , the results for various values of $N$ in Table 1 


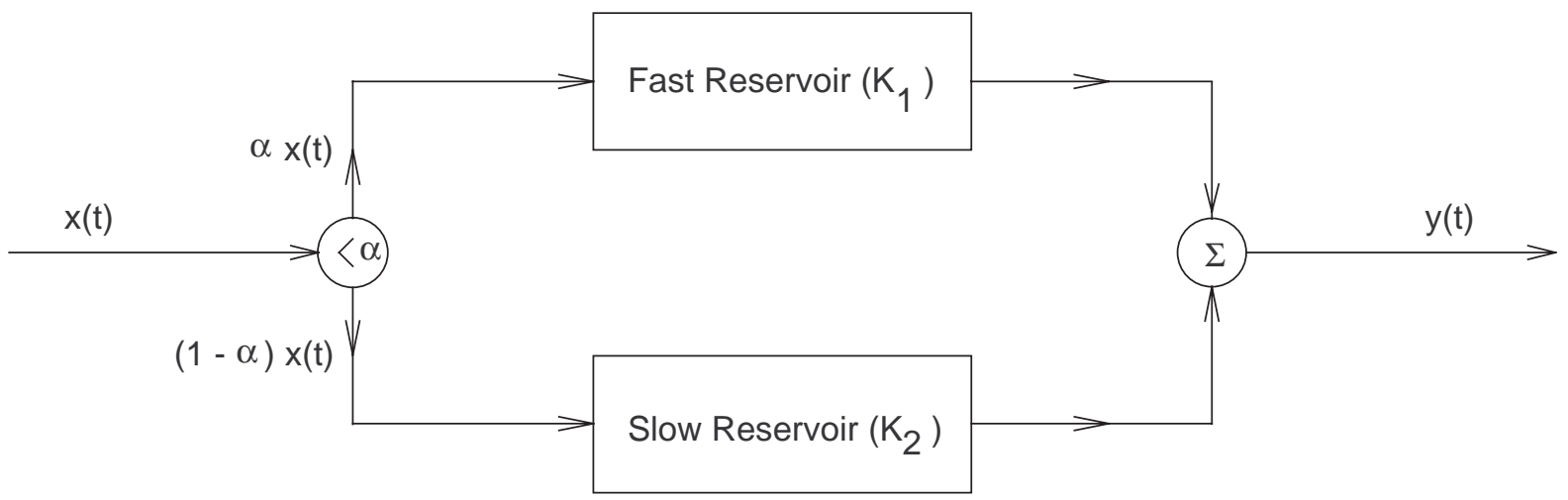

Fig. 3. Simple two-component catchment model

Table 1. Absolute value of the real negative root for two single reservoirs in parallel for different $\alpha$ and timestep $N\left(K_{1}=5, K_{2}=20\right)$.

\begin{tabular}{rlllll}
\hline $\mathrm{N}$ & \multicolumn{5}{c}{$\left|z^{-1}\right|$} \\
& $\begin{array}{l}\alpha=0 \\
\left(K_{2} \text { only }\right)\end{array}$ & $\alpha=0.25$ & $\alpha=0.5$ & $\alpha=0.75$ & $\begin{array}{l}\alpha=1.00 \\
\left(K_{1} \text { only }\right)\end{array}$ \\
\hline 2 & 1.051 & 1.142 & 1.183 & 1.206 & 1.221 \\
4 & 1.051 & 1.136 & 1.178 & 1.204 & 1.221 \\
10 & 1.051 & 1.118 & 1.161 & 1.194 & 1.221 \\
20 & 1.051 & 1.095 & 1.132 & 1.170 & 1.221 \\
40 & 1.051 & 1.075 & 1.096 & 1.122 & 1.221 \\
80 & 1.051 & 1.063 & 1.076 & 1.102 & 1.221 \\
\hline
\end{tabular}

show that the value of $\left|z^{-1}\right|$ is larger for small values of $N$, i.e. it decreases as $N$ increases. Figure 4 presents the Argand diagrams for the cases $N=40$, and $\alpha=(0$ and 0.25$)$ and Fig. 5 for $\alpha=0.5$ and $N=(20$ and 40$)$. The latter shows that increasing $\mathrm{N}$ adds more points to essentially the same circle.

The problem addressed here is to estimate the values of the model parameters, $K_{1}, K_{2}$ and $\alpha$ from a series of solutions of the above type. This could be attempted using a generalpurpose optimisation algorithm to estimate values for the three parameters simultaneously. However, very often in such cases a large number of combinations of parameter values give similar model outputs, which may cause problems for automatic parameter estimation algorithms. The method described here concentrates on identifying physically realistic values for each of the parameters in turn. It is easier to estimate the value of the slow reservoir parameter, $K_{2}$, than the fast, $K_{1}$, because the slow component dominates a greater length of the total runoff hydrograph than the fast. Accordingly, the first step in estimating the system parameters should be to estimate the slow component. It can then be separated from the total hydrograph and the parameter of the quick response can be estimated from the residual hydrograph of shorter duration.
2 single reservoirs in parallel, alpha $=0, N=40$

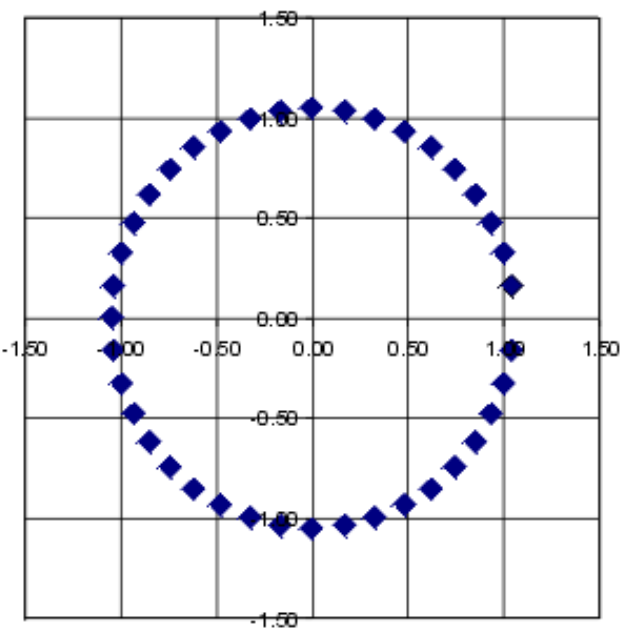

2 single reser voirs in par allel, alpha $=0.25, N=40$

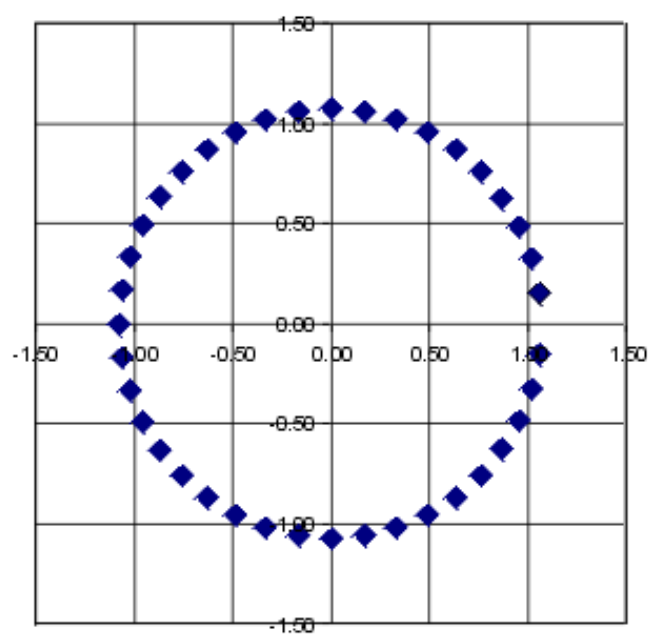

Fig. 4. Roots for two single reservoirs in parallel $\left(K_{1}=5, K_{2}=20\right.$, $\mathrm{N}=40, \alpha=0,0.25$ ) 
2 single reservoirs in parallel, alpha=0.5, $N=20$

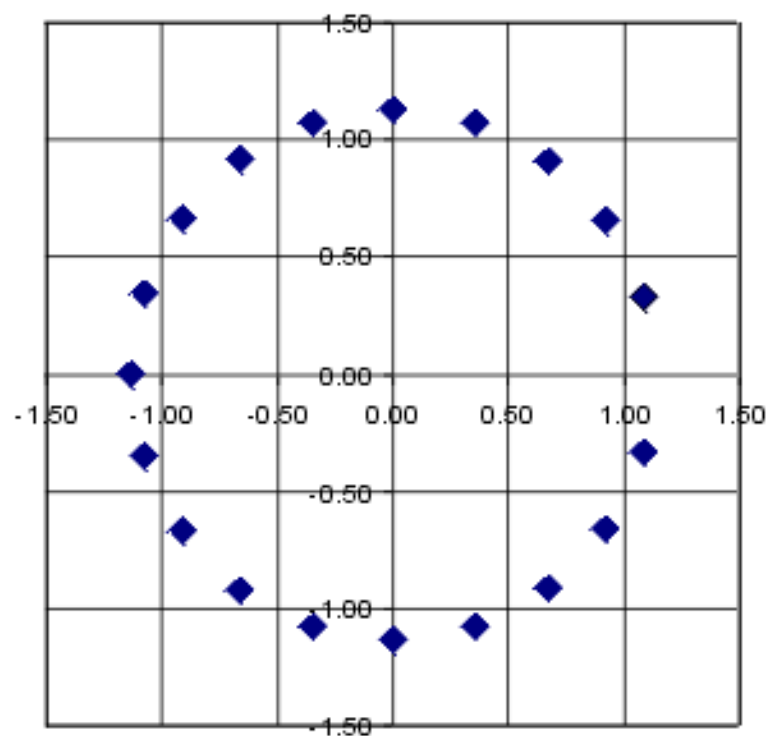

2 single reservoirs in parallel, alpha $=0.5, N=40$

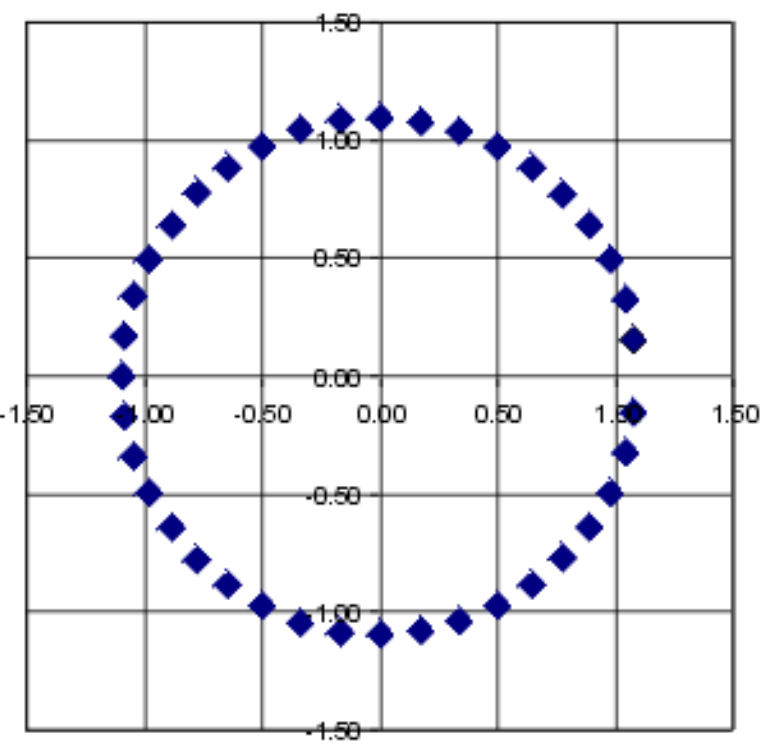

Fig. 5. Roots for two single reservoirs in parallel (, , $\mathrm{N}=20,40$, $a=0.5$ )

\section{Estimation of the slow reservoir parameter}

An estimate of $K_{2}$, denoted $\hat{K}_{2}$, can be calculated by using a high value of $N$. As before, the numerator of Eqn. (8) can be set to zero, i.e.

$$
\mathfrak{I}\left[H\left(z^{-1}\right)\right]=0
$$

Substituting Eqn. (9) and rearranging gives

$$
\left[A_{1} \exp \left(-N T / K_{1}\right)+A_{2} \exp \left(-N T / K_{2}\right)\right]\left(z^{-1}\right)^{N}=A_{1}+A_{2}
$$

For high values of $N$, since $K_{2}>K_{1}, \exp \left(-N T / K_{1}\right)$ will become negligible compared with $\exp \left(-N T / K_{2}\right)$ and this will more than compensate for the fact that $A_{1}>A_{2}$. For values of $N$ of the order of 100 and higher the first term in brackets on the left hand side can be neglected and the equation rewritten as:

$$
A_{2} \exp \left(-N T / K_{2}\right)\left(z^{-1}\right)^{N}=A_{1}+A_{2}
$$

or in the form:

$$
z^{-1}=\left(1+\frac{A_{1}}{A_{2}}\right)^{1 / N} \exp \left(T / K_{2}\right)
$$

If $N$ is sufficiently large, $z^{-1}$ converges to $\exp \left(T / K_{2}\right)$ and thus gives an estimate of the parameter $K_{2}$. Unfortunately, the values of $N$ estimated for typical cases can be in excess of 100 and rounding error can become significant when estimating the polynomial roots using standard precision arithmetic.

Since, in practice, it is not feasible to solve an equation of order 1000, an approximation could be obtained for $N=10$ and $N=100$. Taking $N_{1000}-N_{100}=N_{100}-N_{10}$ which for values of $T=1, K_{1}=5, K_{2}=20$ and $\alpha=0.5$ would give the upper bound of $K_{e q}=23.7$, and a solution sought between $\hat{K}_{2}=15.2$ and $\hat{K}_{2}=23.7$. The average of these estimates is $\hat{K}_{2}=19.45$.

\section{Estimation of the fast reservoir parameter, $K_{1}$}

To estimate the parameter of the fast reservoir, the beginning of the hydrograph is addressed. For the simplest case, taking the first two ordinates only, i.e. $N=2$, there are two real roots and the negative one can be shown to be a close approximation to the root for the fast reservoir in isolation.

For $N=2$, Eqn. (8) becomes:

$$
\begin{aligned}
& H\left(z^{-1}\right)=\alpha\left[\frac{\exp \left(T / K_{1}\right)-1}{T}\right] \exp \left(-T / K_{1}\right) z^{-1}\left[1+\exp \left(-T / K_{1}\right) z^{-1}\right] \\
& \quad+(1-\alpha)\left[\frac{\exp \left(T / K_{2}\right)-1}{T}\right] \exp \left(-T / K_{2}\right) z^{-1}\left[1+\exp \left(-T / K_{2}\right) z^{-1}\right]
\end{aligned}
$$

or

$$
H\left(z^{-1}\right)=B_{1}\left[1+\exp \left(-T / K_{1}\right) z^{-1}\right]+B_{2}\left[1+\exp \left(-T / K_{2}\right) z^{-1}\right]
$$


where:

$$
\begin{aligned}
& B_{1}=\alpha\left[\frac{\exp \left(T / K_{1}\right)-1}{T}\right] \exp \left(-T / K_{1}\right) z^{-1} \\
& B_{2}=(1-\alpha)\left[\frac{\exp \left(T / K_{2}\right)-1}{T}\right] \exp \left(-T / K_{2}\right) z^{-1}
\end{aligned}
$$

Setting $H\left(z^{-1}\right)$ to zero and solving for the roots gives

$$
\begin{aligned}
& z^{-1}=-\frac{B_{1}+B_{2}}{B_{1} \exp \left(-T / K_{1}\right)+B_{2} \exp \left(-T / K_{2}\right)} \\
& =-\frac{1+B_{2} / B_{1}}{1+\left(B_{2} / B_{1}\right) \exp \left(T / K_{1}-T / K_{2}\right)} \exp \left(T / K_{1}\right)
\end{aligned}
$$

For example, for $T=1$ and $K_{1}=5, \mathrm{~B}_{1}=0.051: \alpha$ For $T=1$ and $K_{2}=20, \mathrm{~B}_{2}=0.221 \alpha$ $\alpha=0.5$

$\exp \left(T / K_{1}-T / K_{2}\right)=1.162$

$z^{-1}=-(1.231 / 1.268) \exp \left(T / K_{1}\right)=1.186$

which indicates an equivalent value of $K$, noted $K_{e q}$, as: $\exp \left(T / K_{e q}\right)=1.186$, whence $K_{e q}=5.9$, which is the estimate of $K_{1}$, denoted $\hat{K}_{1}$.

\section{Estimation of the splitting parameter $\alpha$}

If an estimate of the lag between input and output, $U_{1}^{\prime}$, is available from a comparison of measured rainfall with discharge data or from some empirical formula, e.g. in terms of the catchment characteristics, then:

$$
\alpha \hat{K}_{1}+(1-\alpha) \hat{K}_{2}=U_{1}^{\prime}
$$

so that:

$$
\alpha=\frac{\hat{K}_{2}-U_{1}^{\prime}}{\hat{K}_{2}-\hat{K}_{1}}
$$

In the above example, $U_{1}^{\prime}=0.5\left(K_{1}+K_{2}\right)=12.5$.

Accordingly, the use of the estimate

$\hat{K}_{1}=5.9$ and $\hat{K}_{2}=19.5$ would give: $\alpha=\frac{19.5-12.5}{19.5-5.9}=0.51$

which is close to the value of $\alpha=0.50$ used in the simulated data.

\section{Refinement of the initial estimates}

When preliminary estimates of the rainfall pattern and of values of $\mathrm{K}_{1}$ and $\mathrm{K}_{2}$ have been made, these can be improved by an iterative approach. This is analogous to the application of iteration by Collins(1939) to unit hydrograph derivation and by Duband et al. (1993) to the enhancement of the fast component of the total response.

When the total response has been divided into the quick response and the slow response, each can be examined to see if the representation can be improved by relaxing the assumption of $n=1$. For a single reservoir $(n=1)$, the root pattern is a single circle and the value of the negative real root is $z^{-1}=-\exp (T / K)$. For integer values of $n>1$, there is a root at the origin. Where such a root occurs, the best assumption based on experience would be to take $n=2$. For $n=3$, there will be three roots $0,+1$ and $-1 / 2$ so that $z^{-1}=-1 / 2: \exp (T / K)$.

\section{Enhancement of the slow response}

The relative contribution of the quicker component can be enhanced by forward differencing the outflow hydrograph and then analysing the resulting time-series. However, this tends to enhance any errors in the data at the expense of the underlying information. In contrast, auto-correlation, which enhances the slow component, reduces the effect of any data errors by smoothing the total response.

The $K_{2}$ component in the catchment response can be amplified and the effect of measurement noise greatly reduced by using the autocorrelation $\rho(\tau)$ of the output $y(t)$ as the subject of analysis,

$$
\rho(\tau)=\int_{0}^{\infty} y(t) y(t+\tau) d \tau
$$

where, $\tau$ is a time displacement parameter. In the present case, the output is the unit-hydrograph $h(t)$ :

$$
y(t)=h(t)=\alpha a \exp (-a t)+(1-\alpha) b \exp (-b t)
$$

where, $a=T / K_{1}$ and $b=T / K_{2}$. Then:

$$
\begin{aligned}
\rho(\tau)= & {\left[\frac{\alpha^{2}}{2}+\frac{\alpha(1-\alpha) b}{a+b}\right] a \exp (-a \tau)+} \\
& {\left[\frac{\alpha(1-\alpha) a}{a+b}+\frac{(1-\alpha)^{2}}{2}\right] b \exp (-b \tau) }
\end{aligned}
$$

For the example of $\alpha=0.5, \alpha=T / K_{1}=0.2$ and $b=T / K_{2}=0.025$, this gives:

$$
\begin{array}{r}
\rho(\tau)=\frac{1}{4}\left[\frac{1}{2}+\frac{b}{a+b}\right] a \exp (-a \tau)+ \\
\frac{1}{4}\left[\frac{a}{a+b}+\frac{1}{2}\right] b \exp (-b \tau)
\end{array}
$$

which, compared with Eqn. (21), increases the weighting of $b \exp (-b \tau)$ from 0.5 to 0.65 or almost to $2: 1$.

A further autocorrelation would produce a weighting of 0.82 which would further increase the estimate of $K_{2}$ to 18 , compared to the 'true' value of 20 . 


\section{Application to flash floods}

In applying the approach to actual floods, advantage can be taken of the fact that the changes in the state of the system due to the rainfall, the surface processes and the subsurface processes are each dominant in different parts of the hydrograph. Figure 6 shows the hydrograph, sampled at half hourly time steps, for a flash flood on 3 July 1992 in the upper River Dodder $\left(113 \mathrm{~km}^{2}\right)$ which flows through urbanised areas of Dublin, Ireland. A rainstorm which averaged $15 \mathrm{~mm}$ over the catchment, produced a peak flow of $14 \mathrm{~m}^{3} \mathrm{~s}^{-1}$. Figure 7 shows the corresponding root pattern. Selection of the six inner roots as rainfall roots and their inversion to the original time domain gives the estimated rainfall pattern of Fig. 8. The root pattern of Fig. 7 can be separated out as follows. If the rising hydrograph is taken for ordinates 1 to 6 only, the pattern of Fig. 9 is obtained which approximates to, but is not exactly equal to, the inner roots shown for the total response, Fig. 7. The remainder of the hydrograph can be split into two distinct parts - an initial steep recession followed by a much slower one. The segment of the total hydrograph from ordinates 8 to 14 gives the five roots shown on Fig. 10, which lie close to a circle of radius 1.2458 equivalent to $\mathrm{K}_{1} / \mathrm{T}$ approximately equal to 4 ; this, because of the half hour data time step, indicates a single reservoir of lag time $K_{1}=2$ hours as a good approximation. The later part of the hydrograph, from ordinates 22 to 85 gives rise to the root pattern shown in Fig. 11 which is very close to a circle of radius 1.0135 equivalent to $\mathrm{K}_{2} / \mathrm{T}$ approximately equal to 74 , indicating a single reservoir of 37 hours lag time as an excellent approximation. Further refinement is unnecessary. The model of two single

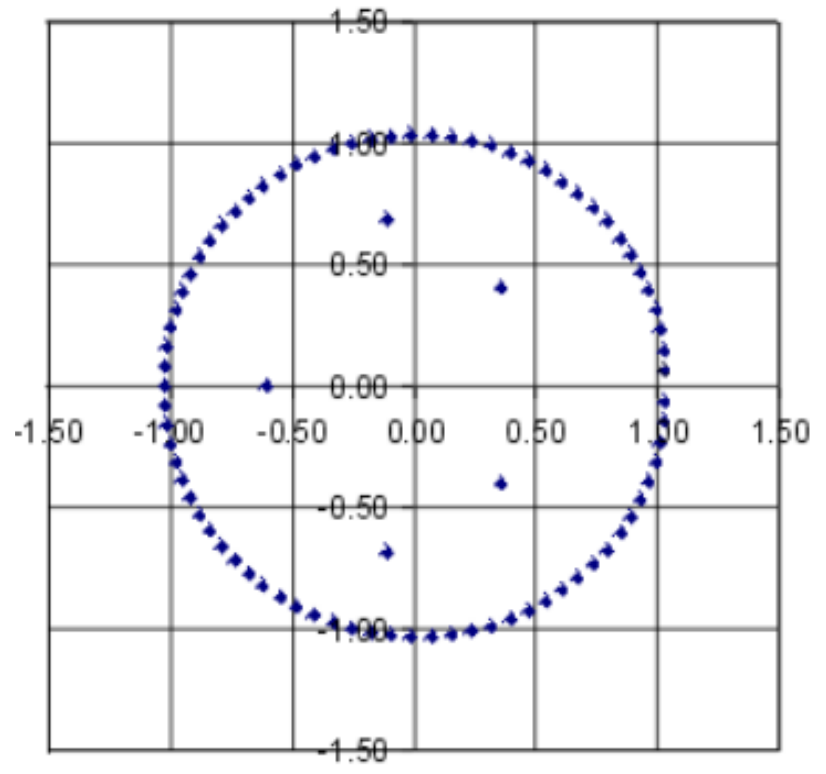

Fig. 7. Root pattern for Dodder flood

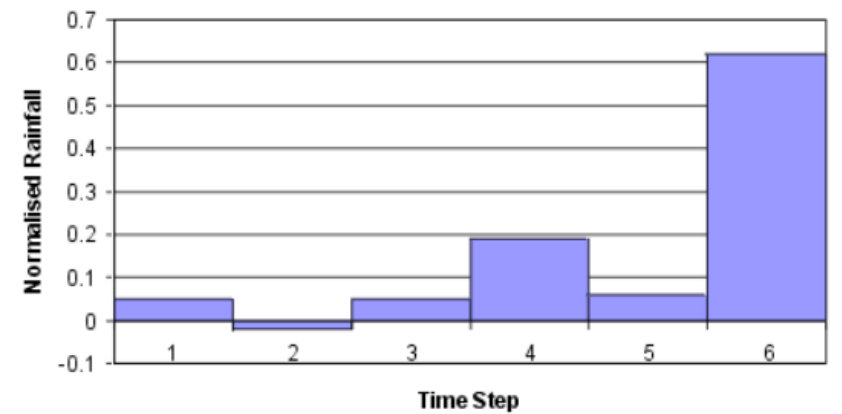

Fig. 8. Estimated rainfall pattern for Dodder flood

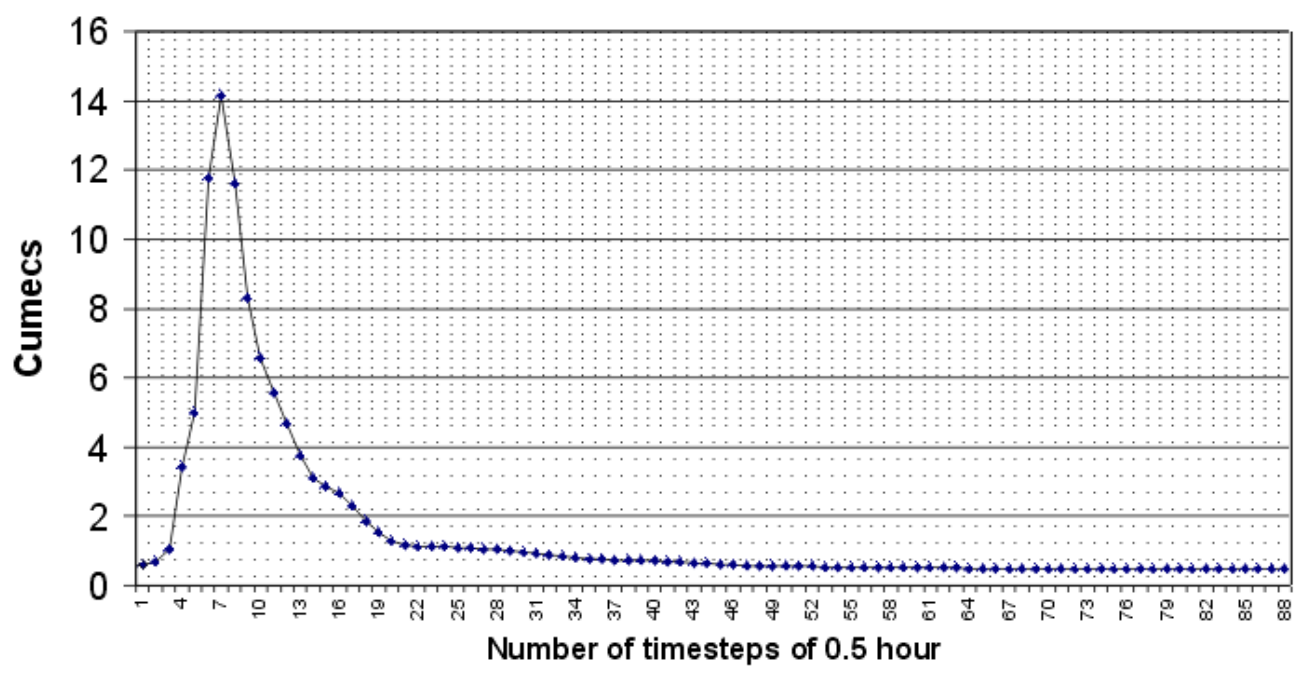

Fig. 6. Hydrograph for the river Dodder flood 


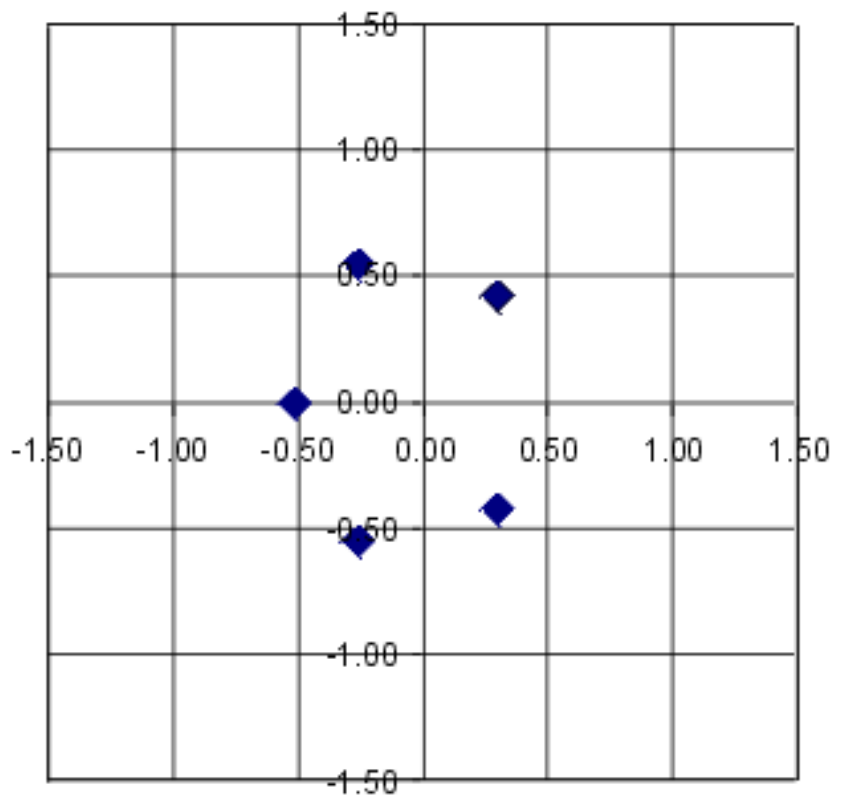

Fig. 9. Roots for ordinates 1 to 6

reservoirs can be adopted or the root patterns shown in Figs. 10 and 11 can be inverted to the time domain.

\section{Conclusion}

This study has developed a reliable method of unithydrograph derivation for flood forecasting from events for which the estimates of effective rainfall are either unreliable or non-existent. Such a method depends on the extraction

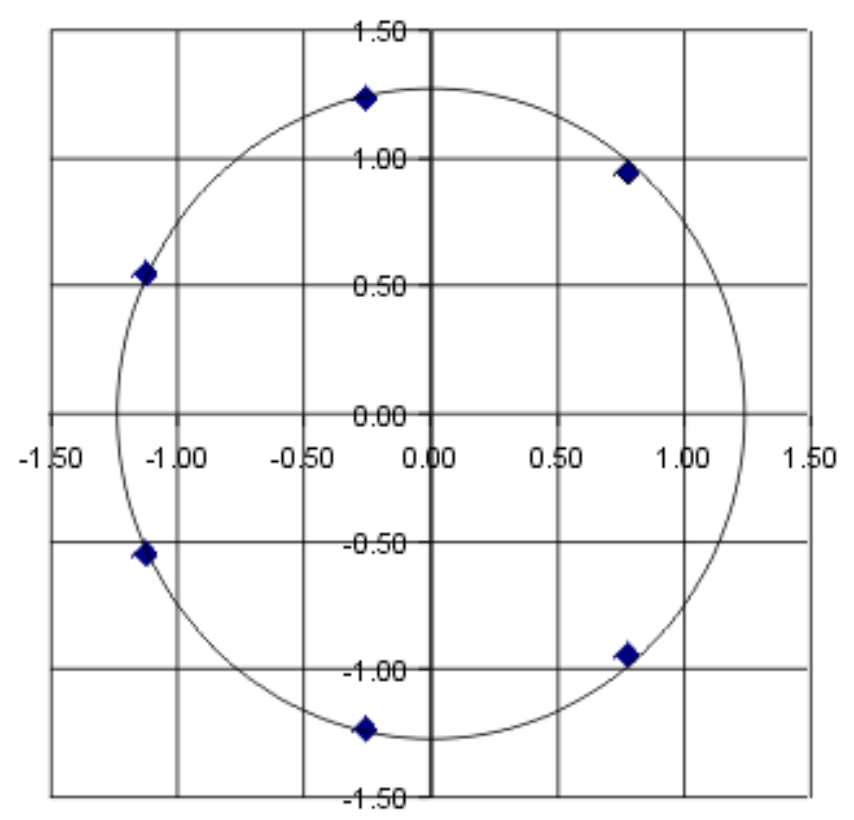

Fig. 10. Roots for ordinates 8 to 14

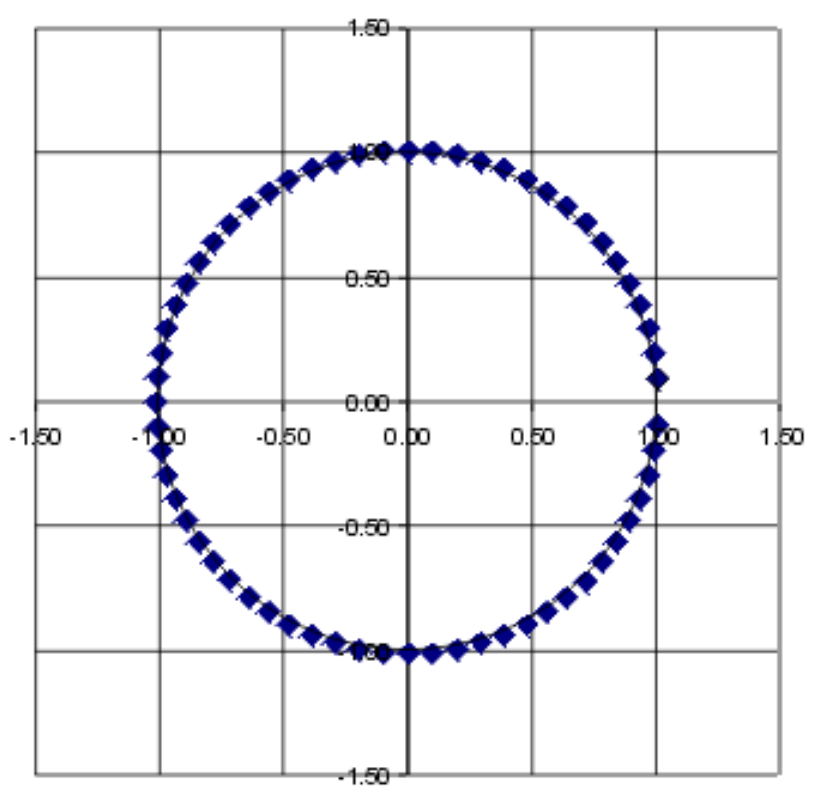

Fig. 11. Roots for ordinates 22 to 85

of an estimated unit-hydrograph solely from the data for catchment runoff.

For the case of sampling interval $T=1$ and a linear reservoir, with a lag time of $K$, analysis indicates a z-transform root pattern on the Argand diagram of a perfect circle with a radius of $\exp (T / K)$. For the case of two linear reservoirs $\left(K_{1}\right.$ and $\left.K_{2}\right)$ in parallel with a proportion $\alpha$ of the total flow through the fast response reservoir $K_{1}$, numerical experiments were conducted for various parameter values. For the values of $\alpha$, other than 0 or 1 , the root pattern no longer forms a perfect circle.

In the case of parallel response functions, the slower response signal can be enhanced by taking the autocorrelation function of the output data. For the synthetic example shown, with $\alpha=0.5$, a single use of autocorrelation increases the proportion of flow through the slower response from $50 \%$ to $65 \%$. A second autocorrelation would increase the proportion further from $65 \%$ to $82 \%$. The use of autocorrelation on real data should smooth out the effect of data errors.

The experience of these numerical experiments on synthetic data was then applied to a single storm event on the Dodder catchment. The z-transform root pattern was shown for this data set and its slow and fast components were identified. The work described above represents a new step in the development of the root-matching-root selection approaches, due to de Laine and Turner respectively. To distinguish the method described here from the previous versions of this general approach, it is described as the 'root separation method'. 
Earlier in the paper, the four disadvantages of the original root-matching method of de Laine (1970) as described by Turner et al. (1989) were quoted. The first two disadvantages are overcome by the root selection method (Turner 1982, 1989) since the shift in the actual values of the polynomial does not affect the overall pattern. A difficulty remains in the case of uniform effective rainfall or an extremely U-shaped distribution of rainfall, both of which give rise to a circular pattern of rainfall roots which may not be readily separated from the response roots for a single reservoir. The third disadvantage, effects of choice of method of base flow separation, is overcome by separating the root pattern of the total response into its component parts. In the example given, the reduction of total rainfall to effective rainfall is on the basis of a single coefficient. This can be extended to other forms of separation, but this question is not addressed here. In regard to the fourth disadvantage, the assumption of linearity, there is the possibility of extending the approach to the case of non-linearity (Dooge, 1967) but this also is outside the scope of the present paper.

\section{Acknowledgments}

The authors are grateful for the helpful comments and suggestions of the anonymous reviewers. The work was made possible by the support of the European Commission, through the following research projects:

1. TELFLOOD Project: Forecasting Floods in Urban Areas Downstream of Steep Catchments (ENV4CT96-0257). 1999.

2. CARPE DIEM Project: Critical assessment of Available Radar Precipitation Estimation techniques and Development of Innovative approaches for Environmental Management (EVG1-2001-00031). In progress.

\section{References}

Collins, W.T., 1939. Runoff distribution graphs for precipitation occurring in more than one time unit. Civil Eng., 9, 559-561.

de Laine, R.J., 1970. Deriving the unit-hydrograph without using rainfall data. J. Hydrol., 10, 379-390.

de Laine, R.J., 1975. Identifying a time-invariant system from its inputs. $\mathrm{PhD}$ thesis, Monash University, Victoria, Australia.

Dooge, J.C.I., 1959. A general theory of the unit-hydrograph. $J$. Geophys. Res., 64, 241-256.

Dooge, J.C.I., 1967. A new approach to non-linear problems in surface water hydrology. Proc. IAHS General Assembly, Bern. 409-413.

Dooge, J.C.I., 1973. Linear theory of hydrologic systems. Technical Bulletin No. 1468, 327pp. U.S. Agricultural Research Service, Washington, USA.

Dooge, J.C.I., 1979. Deterministic input-output models. In: The Mathematics of Hydrology and Water Resources, E.H. Lloyd, T. O'Donnell and J.C. Wilkinson (Eds.). Academic Press, London, UK.

Duband, C., Obled, C. and Rodriguez, J.Y., 1993. Unit-hydrograph revisited: an alternative iterative approach to unit-hydrograph and effective rainfall identification. J. Hydrology, 150, 115149.

Eagleson, P.S., Mejia-R., R. and March, F., 1966. Computation of optimum realizable unit-hydrographs. Water Resour. Res., 2, 755-764.

Jenkins, M.A. and Traub, J.F., 1972. Zeros of a complex polynomial, algorithm no. 419. Comm. Assoc. Computing Machinery, 15, 97.

Johnstone, D. and Cross, W.P., 1949. Elements of Applied Hydrology. Ronald Press, New York, USA.

Nash, J.E., 1958. The form of the instantaneous hydrograph. IASH Gen. Ass. Toronto, IAHS Publ. No. 42, 3: 114-118.

O'Connor, K.M., 1976. A discrete linear cascade for hydrology. J. Hydrol., 29, 203-242.

O'Connor, K.M., 1982. Derivation of discretely coincident forms of continuous linear time-invariant models using the transfer function approach. J. Hydrol., 59, 1-48.

Turner, J.E., 1982. A real-time flood forecasting model for the River Liffey. Master's thesis, University College Dublin, Ireland.

Turner, J.E., Dooge, J.C.I. and Bree, T., 1989. Deriving the unithydrograph by root selection. J. Hydrol., 110, 137-152. 


\section{Appendix: List of symbols and notations}

\author{
$x(t), y(t), h(t)$ \\ $x(s T), y(s T), h(s T)$ \\ $X(p, n)$ \\ $\boldsymbol{x}(m)$ \\ $\boldsymbol{y}(p)$ \\ $\boldsymbol{h}(n)$ \\ (n) \\ $\mathrm{m}$ \\ $p$
}

Unit-hydrograph theory

\section{Root Methods}

$T, s T$

$t$

$K, K_{1}, K_{2}, K_{e q}$

$\alpha$

$n$

$x(t), y(t), h(t)$

$x(s T), y(s T), h(s T)$

N

$F\left(z^{-1}\right)$

$X\left(z^{-1}\right), Y\left(z^{-1}\right), H\left(z^{-1}\right)$

$\mathrm{U}_{1}^{\prime}$

$\mathfrak{P}^{(\tau)}$ continuous input, output and transfer functions

discrete input, output and transfer functions

matrix of input made of the effective rainfall

input vector, i.e. the effective rainfall

output vector, i.e. the direct runoff

transfer function vector

estimate of $\boldsymbol{h}(n)$

length of the input time series

length of the output time series

discrete timestep, discrete time variable

continuous time variable

reservoir parameters ( $\hat{K}$ denotes the estimate of $K$ )

parameter for the splitting of input into a system of reservoirs in parallel

number of reservoirs in series

continuous input, output and transfer functions

discrete input, output and transfer functions

length of a unit hydrograph or number of ordinates in a hydrograph time-series

selected to derive the $\mathrm{z}$-transform

$\mathrm{z}$-transform of the discrete function $f(s t)$

z-transforms of the input, output and transfer functions

lag between input and ouptut

autocorrelation function for lag $\mathrm{t}$

the numerator of the z-transform, 
